\title{
Diagnostic Tool for Assessing Overactive Bladder Symptoms: Could the International Prostate Symptom Storage Subscore Replace the Overactive Bladder Symptom Score?
}

\author{
Ji Sung Shim¹, Jae Heon Kim², Hoon Choi ${ }^{1}$, Jae Young Park ${ }^{1}$, Jae Hyun Bae \\ ${ }^{1}$ Department of Urology, Korea University Ansan Hospital, Korea University College of Medicine, Ansan, Korea \\ ${ }^{2}$ Department of Urology, Soonchunhyang University Hospital, Soonchunhyang University College of Medicine, Seoul, Korea
}

Purpose: The goal of this study was to compare the International Prostate Symptom Storage Subscore (IPSS-s) and the overactive bladder symptom score (OABSS) as tools for assessing the symptoms of overactive bladder (OAB).

Methods: A cross-sectional study was conducted of a sample of 1,341 patients aged 50 years and older with lower urinary tract complaints who had undergone a medical examination at one of several centers. For each patient, we reviewed the International Prostate Symptom Score and the OABSS. The patients were divided into 2 groups according to their IPSS-s result (group 1 , score $\geq 6$; group 2, score $<6$ ) and into another 2 groups according to their OABSS diagnosis (group 3, OAB patients; group 4 , non-OAB patients). We determined whether the OABSS varied to a statistically significant extent between groups 1 and 2 . Furthermore, we evaluated the correlation of IPSS-s severity with the OABSS results in group 3, and the OAB diagnosis rate was compared between groups 1 and 2 .

Results: In groups 1 and 2, the OABSS results were not found to vary to a statistically significant extent $(\mathrm{P}=0.326)$. In group 3 , no significant correlation was found between IPSS-s severity and the OABSS results $(\mathrm{P}=0.385)$. In the prevalence analysis, no statistically significant difference was found among the groups, and the receiver operating characteristic curve showed an area under the curve of 0.474 .

Conclusions: The results of this cross-sectional analysis suggest that the IPSS-s and the OABSS are not significantly correlated. Although both scores are used to measure OAB symptoms, the simultaneous use of IPSS-s and OABSS is not warranted.

Keywords: Diagnosis; Lower Urinary Tract Symptoms; Urinary Bladder, Overactive; Prevalence; Surveys and Questionnaires

- Research Ethics: This study was conducted in accordance with the ethical standards laid down in the 1964 Declaration of Helsinki and its later amendments, and the appropriate ethical review boards approved this retrospective study (AS13030).

- Conflict of Interest: No potential conflict of interest relevant to this article was reported.

\section{INTRODUCTION}

Overactive bladder $(\mathrm{OAB})$ is defined as urinary urgency, with or without urge incontinence and usually with frequency and nocturia, in the absence of an infection or other obvious patho- logic conditions [1]. Since OAB is defined by subjective rather than objective symptoms, the patient's perspective is important in managing this disease.

For this reason, several diagnostic tools evaluating patientreported outcomes are available. The overactive bladder symp-

Corresponding author: Jae Hyun Bae (D) http://orcid.org/0000-0001-9862-3545 Department of Urology, Korea University Ansan Hospital, Korea University College of Medicine, 123 Jeokgeum-ro, Danwon-gu, Ansan 15355, Korea E-mail: urobae@genetherapy.or.kr / Tel: +82-31-412-5190 / Fax: +82-31-412-5194 Submitted: February 15, 2016 / Accepted after revision: April 4, 2016 
tom score (OABSS) is based on a validated self-assessment questionnaire consisting of 4 questions assessing $\mathrm{OAB}$ symptoms. Patients respond to each question about their bladder symptoms during the previous week. The OABSS provides an overall composite score, as well as individual scores [2].

However, the International Prostate Symptom Score (IPSS) is the most commonly used assessment tool for men with lower urinary tract symptoms (LUTS) [3,4]. The IPSS consists of a total of 7 questions on voiding symptoms (incomplete emptying, intermittency, weak stream, and straining to void) and storage symptoms (frequency, urgency, and nocturia).

Generally, many clinicians employ the IPSS to assess patients complaining of LUTS. Moreover, when the chief complaint is urgency or urge incontinence, clinicians tend to investigate $\mathrm{OAB}$ using the International Prostate Symptom Storage Subscore (IPSS-s) [5]. However, the relationship of the OABSS to the IPSS has not been sufficiently evaluated. It is not unusual for the IPSS-s alone to be used for assessing OAB. In the present study, we compared the IPSS-s and the OABSS as tools for assessing $\mathrm{OAB}$.

\section{MATERIALS AND METHODS}

\section{Patient Sample and Data Collection}

A cross-sectional retrospective study was conducted, with a total sample of 1,341 patients. We enrolled patients older than 50 who voluntarily underwent a free medical checkup at the Health Promotion Center in Seoul from 2012 to 2014. The following inclusion criteria were used: patient age older than 50 years, good overall mental and physical health, and LUTS complaints. We reviewed the IPSS and OABSS results for each patient. The patients were divided into 2 groups according to their IPSS-s result (group 1, score $\geq 6$; group 2, score $<6$ ) and into another 2 groups according to their OAB diagnosis (group 3, OAB patients; group 4, non-OAB patients) based on the OABSS criteria [6].

\section{Assessment Tools}

\section{International Prostate Symptom Score}

The IPSS was adapted from the American Urological Association symptom index and quality of life questionnaire and has emerged as the standard tool for the evaluation of symptoms of benign prostatic hyperplasia (BPH) [3]. The symptom index consists of 7 questions on incomplete emptying, urinary frequency, intermittency, urgency, weak urinary stream, hesitancy, and nocturia. In this tool, a total score of $0-35$ is calculated by summing the scores of $0-5$ for the 7 questions. The quality of life index is scored on a scale of $0-6$, according to the patient's symptoms.

\section{Overactive Bladder Symptom Score}

The OABSS is based on a symptom assessment questionnaire designed to quantify $\mathrm{OAB}$ symptoms as a single score, and contains questions about the following 4 symptoms of $\mathrm{OAB}$ : daytime frequency, nighttime frequency, urgency, and urge incontinence. In this tool, the patients are asked to rate their symptom severity on a Likert scale with the maximum (worst) scores of $2,3,5$, and 5 . The total score ranges from 0 to 15 , and a more severe $\mathrm{OAB}$ is indicated by a higher score. The scoring system is designed to place more weight on urgency and urgency incontinence than on frequency. The questionnaire has been shown to satisfy psychometric criteria for reliability [2].

\section{Statistical Analysis}

Continuous variables were expressed as the mean \pm standard deviation, median (interquartile range), or as the number (percentage) of cases. Categorical variables were reported as the number and percentage. The Student t-test and Spearman correlation coefficient were used for the statistical comparisons of categorical and continuous variables, respectively. The chisquare test was used to investigate the prevalence of various conditions between groups. Furthermore, receiver operating characteristic (ROC) curves were used to assess the ability of the IPSS-s to predict OAB diagnoses according to the OABSS diagnostic criteria. All analyses were performed with IBM SPSS Statistics ver. 20.0 (IBM Co., Armonk, NY, USA). P-values $<0.05$ were considered to indicate statistical significance.

\section{RESULTS}

The patients' mean age was $73.9 \pm 7.6$ years, and their age distribution is presented in Table 1 . The mean total IPSS, IPSS-voiding subscore, IPSS-s, and OABSS results were 4.8 $\pm 7.7,2.6 \pm 4.7$, $2.2 \pm 3.4$, and $3.2 \pm 3.5$, respectively.

The results of the comparison of OABSS results between groups 1 and 2 (defined according to their IPSS-s results) are shown in Table 2 (Student t-test). None of the 4 questions of the OABSS questionnaire showed a significant result; similarly, the total OABSS did not significantly differ between these 2 groups $(\mathrm{P}=0.326)$. Additionally, we compared urgency symptom 
Table 1. Baseline patient characteristics

\begin{tabular}{ll}
\hline Variable & Value \\
\hline Age $(\mathrm{yr})$ & $73.9 \pm 7.6$ \\
$50-60$ & $109(8.12)$ \\
$61-70$ & $366(27.29)$ \\
$71-80$ & $647(48.25)$ \\
$>80$ & $219(16.33)$ \\
Sex & \\
Male & $454(33.86)$ \\
Female & $887(66.14)$ \\
Total IPSS & $4.8 \pm 7.7$ \\
IPSS voiding subscore & $2.6 \pm 4.7$ \\
IPSS storage subscore & $2.2 \pm 3.4$ \\
Total OABSS & $3.2 \pm 3.5$ \\
\hline
\end{tabular}

Values are presented as mean \pm standard deviation or number of patients (\%).

IPSS, International Prostate Symptom Score; OABSS, overactive bladder symptom score.

Table 2. Results of the comparison between the 2 groups according to the IPSS-s of the OABSS questions

\begin{tabular}{lccc}
\hline OABSS question & IPSS-s $\geq 6$ & IPSS-s $<6$ & P-value \\
\hline Q1 & $0.3 \pm 0.5$ & $0.3 \pm 0.6$ & 0.407 \\
Q2 & $1.2 \pm 2.5$ & $1.4 \pm 1.1$ & 0.092 \\
Q3 & $0.9 \pm 1.4$ & $0.9 \pm 1.4$ & 0.813 \\
Q4 & $0.6 \pm 1.1$ & $0.6 \pm 1.2$ & 0.482 \\
Total & $3.0 \pm 4.3$ & $3.3 \pm 3.4$ & 0.326 \\
\hline
\end{tabular}

Values are presented as mean \pm standard deviation.

IPSS-s, International Prostate Symptom Storage subscore; OABSS, overactive bladder symptom score; Q, question.

${ }^{*} \mathrm{P}<0.05$, Student t-test.

scores with the total OABSS, and likewise found no statistical significance $(\mathrm{P}=0.616)$.

Furthermore, the correlation of the IPSS-s severity with OABSS results was investigated in groups 3 and 4 (defined on the basis of $\mathrm{OAB}$ diagnoses). All correlation coefficients were negative or not statistically significant (total OABSS; $\mathrm{P}=0.385, \mathrm{r}=$ -0.048 ) (Table 3).

We evaluated the $\mathrm{OAB}$ diagnosis rate in patients grouped according to their IPSS-s results (score $\geq 6$ vs. score $<6$ ) using the chi-square test, and found no statistically significant difference ( $\mathrm{P}=0.092$; odds ratio, 1.320; 95\% confidence interval, 0.9551.826) (Table 4). Moreover, a ROC curve was constructed to assess the ability of the IPSS-s to predict the diagnosis of OAB according to the OABSS diagnostic criteria. The area under the
Table 3. Correlation of the IPSS-s severity with the OABSS in the $\mathrm{OAB}$ diagnosis group

\begin{tabular}{lcc}
\hline OABSS question & Spearman correlation coefficient & P-value \\
\hline Q1 & -0.008 & 0.880 \\
Q2 & 0.067 & 0.224 \\
Q3 & -0.116 & $0.035^{\star}$ \\
Q4 & -0.042 & 0.444 \\
Total & -0.048 & 0.385 \\
\hline
\end{tabular}

IPSS-s, International Prostate Symptom Storage subscore; OABSS, overactive bladder symptom score; $\mathrm{OAB}$, overactive bladder; $\mathrm{Q}$, question.

${ }^{\star} \mathrm{P}<0.05$.

Table 4. OAB diagnosis rate in patients grouped according to the IPSS-s association on the chi-square test

\begin{tabular}{lcccc}
\hline Group & $\begin{array}{c}\text { OAB } \\
(n=332)\end{array}$ & $\begin{array}{c}\text { Non-OAB } \\
(n=1,009)\end{array}$ & P-value & OR (95\% CI $)$ \\
\hline IPSS-s & & & $0.092^{*}$ & $1.320(0.955-1.826)$ \\
$\geq 6(n=215)$ & $63(29.3)$ & $152(70.7)$ & & \\
$<6(n=1,126)$ & $269(23.9)$ & $857(76.1)$ & & \\
\hline
\end{tabular}

$\mathrm{OAB}$, overactive bladder; IPSS-s, International Prostate Symptom Storage subscore; $\mathrm{OR}$, odds ratio; $\mathrm{CI}$, confidence interval. ${ }^{*} \mathrm{P}<0.05$.

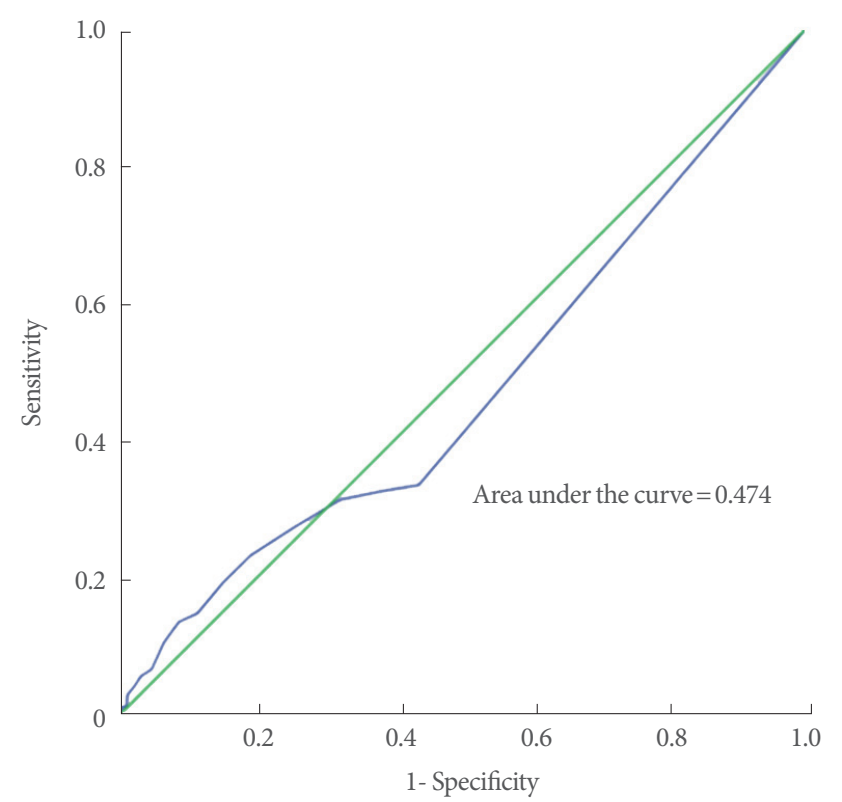

Fig. 1. Receiver operating characteristic (ROC) curves for the ability of International Prostate Symptom Storage subscore to predict the overactive bladder diagnosis rate according to the overactive bladder symptom score diagnosis criteria. The ROC curve showed an area under the curve of $0.474(\mathrm{P}=0.160)$. 
curve was $0.474(\mathrm{P}=0.160)$ (Fig. 1).

\section{DISCUSSION}

This analysis compared the OABSS and the IPSS-s in order to determine whether the IPSS-s can be used as a replacement tool for the OABSS. We found no significant correlation between the IPSS-s and the OABSS, which means that the simultaneous use of the 2 tools should be avoided.

The assessment of LUTS is indispensable for an accurate diagnosis, the proper choice of treatment, and evaluating the efficacy of treatment of lower urinary tract disorders. For patients complaining of LUTS, the IPSS questionnaire has often been used as a symptom assessment tool [7-9]. Many urologists use the IPSS in daily practice in investigations of LUTS in both female and male patients [10-13].

However, although an IPSS-s result of $\geq 6$ is somewhat meaningful, whether it can be used for assessing OAB is still controversial. This may be because the IPSS was originally designed for the assessment of BPH symptoms [3]. Some studies have argued that the IPSS-s can be used for evaluating storage LUTS; however, scant evidence supports the use of the IPSS-s for assessing $\mathrm{OAB}[5]$.

In our study, the patients were divided into 2 groups according to a high or low storage subscore. No significant correlations were found between each question of the OABSS questionnaire and the IPSS-s, or for the total OABSS. The above results may indicate that even a high IPSS-s may not be useful for diagnosing $\mathrm{OAB}$.

Furthermore, we evaluated the correlation between IPSS-S severity and the OABSS, and likewise found no statistically significant results. As discussed above, the composition of the questionnaire is slightly different in these 2 diagnostic tools. Frequency, nocturia, and urgency are common items in both questionnaires, but urge incontinence is only included in the OABSS questionnaire.

Considering the small differences in the questionnaire composition, it was unexpected that the 2 diagnostic tools were not found to be correlated. The OABSS places more weight on the scores for urgency and urgency incontinence (maximum score, 5 ) than on micturition frequency (maximum score, 2 or 3). For $\mathrm{OAB}$ patients, the most bothersome symptom is urgency or perhaps urge incontinence (when the latter symptom is present) $[14,15]$. These considerations may explain the discrepancy between these diagnostic tools.
The OABSS was developed and validated in order to collectively express $\mathrm{OAB}$ symptoms as a single score [16]. Since OAB is a collection of symptoms, evaluating all the symptoms, rather than a single or a limited number of symptoms, may be expected to show better performance in the assessment of symptoms and their changes. Therefore, the reason for sometimes using the IPSS-s instead of the OABSS may be that OAB symptoms have usually been quantified by counting episodes of micturition, urgency, and nocturia. However, the diagnosis rate of $\mathrm{OAB}$ was not significantly higher in the group with a high storage subscore than in the group with a low storage subscore. Moreover, we found the IPSS-s to be an insufficient indicator in diagnosing $\mathrm{OAB}$ due to its relatively low area under the curve (0.474) in the ROC analysis.

This study has some limitations. First, this was a cross-sectional investigation of a Korean population with clinical diagnoses involving a relatively small number of patients. Therefore, our results may not be generalizable to other populations. Second, the current study may have been affected by selection bias. Prospective studies with a larger number of patients are warranted to confirm the present results, which suggest that the IPSS-s cannot replace the OABSS as an assessment tool for OAB, and that the indiscriminate use of both questionnaires should be avoided.

\section{REFERENCES}

1. Abrams P, Cardozo L, Fall M, Griffiths D, Rosier P, Ulmsten U, et al. The standardisation of terminology of lower urinary tract function: report from the Standardisation Sub-committee of the International Continence Society. Am J Obstet Gynecol 2002;187:11626.

2. Homma Y, Kakizaki H, Yamaguchi O, Yamanishi T, Nishizawa O, Yokoyama O, et al. Assessment of overactive bladder symptoms: comparison of 3-day bladder diary and the overactive bladder symptoms score. Urology 2011;77:60-4.

3. Barry MJ, Fowler FJ Jr, O'Leary MP, Bruskewitz RC, Holtgrewe HL, Mebust WK, et al. The American Urological Association symptom index for benign prostatic hyperplasia. The Measurement Committee of the American Urological Association. J Urol 1992;148: 1549-57.

4. Chapple C, Herschorn S, Abrams P, Sun F, Brodsky M, Guan Z. Tolterodine treatment improves storage symptoms suggestive of overactive bladder in men treated with alpha-blockers. Eur Urol 2009;56:534-41. 
5. Hsiao SM, Lin HH, Kuo HC. International Prostate Symptom Score for assessing lower urinary tract dysfunction in women. Int Urogynecol J 2013;24:263-7.

6. Lee JY, Lee SH, Kim SJ, Kim CS, Lee HM, Kim CI, et al. Change in International Prostate Symptom storage subscore after long-term medical therapy in BPH patients: finasteride and alpha-blocker combination therapy in men with moderate-to-severe LUTS/BPH in Korea. Urology 2011;77:171-6.

7. Keyes M, Miller S, Moravan V, Pickles T, McKenzie M, Pai H, et al. Predictive factors for acute and late urinary toxicity after permanent prostate brachytherapy: long-term outcome in 712 consecutive patients. Int J Radiat Oncol Biol Phys 2009;73:1023-32.

8. Moore KN, Valiquette L, Chetner MP, Byrniak S, Herbison GP. Return to continence after radical retropubic prostatectomy: a randomized trial of verbal and written instructions versus therapistdirected pelvic floor muscle therapy. Urology 2008;72:1280-6.

9. Suardi N, Scattoni V, Briganti A, Salonia A, Naspro R, Gallina A, et al. Nerve-sparing radical retropubic prostatectomy in patients previously submitted to holmium laser enucleation of the prostate for bladder outlet obstruction due to benign prostatic enlargement. Eur Urol 2008;53:1180-5.

10. Low BY, Liong ML, Yuen KH, Chee C, Leong WS, Chong WL, et al. Terazosin therapy for patients with female lower urinary tract symptoms: a randomized, double-blind, placebo controlled trial. J
Urol 2008;179:1461-9.

11. Maserejian NN, Kupelian V, McVary KT, Doshi M, Link CL, McKinlay JB. Prevalence of post-micturition symptoms in association with lower urinary tract symptoms and health-related quality of life in men and women. BJU Int 2011;108:1452-8.

12. Okamura K, Nojiri Y, Osuga Y, Tange C. Psychometric analysis of international prostate symptom score for female lower urinary tract symptoms. Urology 2009;73:1199-202.

13. Rosen RC, Link CL, O'Leary MP, Giuliano F, Aiyer LP, Mollon P. Lower urinary tract symptoms and sexual health: the role of gender, lifestyle and medical comorbidities. BJU Int 2009;103 Suppl 3:42-7.

14. Chapple CR, Artibani W, Cardozo LD, Castro-Diaz D, Craggs M, Haab F, et al. The role of urinary urgency and its measurement in the overactive bladder symptom syndrome: current concepts and future prospects. BJU Int 2005;95:335-40.

15. Temml C, Haidinger G, Schmidbauer J, Schatzl G, Madersbacher S. Urinary incontinence in both sexes: prevalence rates and impact on quality of life and sexual life. Neurourol Urodyn 2000;19:25971.

16. Coyne KS, Matza LS, Thompson CL, Kopp ZS, Khullar V. Determining the importance of change in the overactive bladder questionnaire. J Urol 2006;176:627-32. 